\title{
Achondrogenesis Type II in a Live Term Newborn Infant: A Case Report
}

\author{
Mustapha Bello1*, Tope Bello², Ahmadu Baba Usman ${ }^{3}$, Adzu Yusuf ${ }^{3}$, Simon Pius', \\ Harifarta Difirwiti ${ }^{3}$, Yakubu Sani ${ }^{3}$
}

\author{
${ }^{1}$ Department of Paediatrics, University of Maiduguri Teaching Hospital, Maiduguri, Nigeria \\ ${ }^{2}$ Department of Radiology, Ladoke Akintola University of Technology, Ogbomosho, Nigeria \\ ${ }^{3}$ Department of Paediatrics, Federal Medical Centre, Yola, Nigeria \\ Email: ‘bellomustapha@unimaid.edu.ng
}

How to cite this paper: Bello, M., Bello, T. Usman, A.B., Yusuf, A., Pius, S., Difirwiti, H. and Sani, Y. (2018) Achondrogenesis Type II in a Live Term Newborn Infant: A Case Report. Open Journal of Pediatrics, 8, 58-65.

https://doi.org/10.4236/ojped.2018.81008

Received: January 30, 2018

Accepted: March 6, 2018

Published: March 9, 2018

Copyright (C) 2018 by authors and Scientific Research Publishing Inc. This work is licensed under the Creative Commons Attribution International License (CC BY 4.0).

http://creativecommons.org/licenses/by/4.0/

\begin{abstract}
Background: Achondrogenesis type II is a lethal form of osteochondrodysplasia characterized by short trunk, disproportionately large head, prominent forehead, micrognathia, extreme micromelia, anasarca, large abdomen and poor ossification of the bones. The children with achondrogenesis are usually born premature, or die in the neonatal period mostly from respiratory failure. We report the case of a live term newborn infant with achondrogenesis type II who died shortly after birth. Methods: We report a case of achondrogenesis type II in a live male newborn. Results: We report the case of a term male infant delivered to a 24-year-old woman with a chondrogenesis type II confirmed radiologically but died at age 5 days. Conclusion: Whenever a skeletal dysplasia in a fetal dwarfism is suspected, a proper work-up plan should be done to evaluate family history. A clinical, radiographic and histopathologic examination, should be done and confirmed by genetic study. Following evidence-based diagnosis, patients could be offered termination of pregnancy after counseling.
\end{abstract}

\section{Keywords}

Achondrogenesis Type II, Lethal Malformation, Live Born Infant

\section{Introduction}

Achondrogenesis is a very rare group of severe disorder that affects cartilage and bone development [1]. Achondrogenesis was described in 1925 by Donath and Vogl, and was first used in the medical literature in 1952 by an Italian pathologist named Marico Fraccaro [2] [3] [4] [5]. By the 1970s, researchers concluded 
that achondrogenesis was a heterogeneous group of chondroplasia lethal to neonate. Achondrogenesis type I and II were distinguished on the bases of radiological and histological criteria. In 1983, a new radiological classification of achondrogenesis type I-IV by Whitley and Gorlin was adopted in the McKusick criteria [5].

Achodrogenesis was derived from Greek word meaning "not producing cartilage" [2]. It is a lethal disorder characterized by extreme micromelia, short trunk, a disproportionately large cranium, and anasarca [6] [7] [8]. Radiological features are characteristic, with virtual absence of ossification of the vertebral column, sacrum, pelvic bone, poor ossification of the skull, multiple rib fracture (Type IA and Type IB), and very short, broad bones of the extremities with marked bowing [1] [6] [7] [8].

There are two types of Achondrogenesis. Type I is of an autosomal recessive inheritance with subtype IA (Houston-Haris type) and type IB (Parenti-Fraccaro type), and are often hard to tell apart without genetic testing [1] [6] [7] [9]. Type IA is the least well understood one of the three forms of achondrogenesis. It results from mutation in TRIP II gene which provides instructions for making a protein GMAP-210. This protein plays a critical role in Golgi apparatus, a cell structure in which newly produced proteins are modified so they can carry out their function [9], while type IB results from mutation of SLC26A2 gene which provides instructions for making a protein that is essential for the development of cartilage and for its conversion to bone [9].

Achondrogenesis type II (Langer-Saldino) is mostly sporadic and was initially described by Langer et al. and Saldino [1] [10]. Achondrogenesis type II is caused by de novo dominant mutations in collagen type II-I COL2AI gene [1] [6] [7]. A transition of $\mathrm{G}^{2853}$ to $\mathrm{A}$ in axon 41 produced a substitution of $\mathrm{Gly}^{769}$ by Ser within the triple helical domain of the $\alpha \mathrm{I}$ (II) chain type II collagen, interrupting the mandatory Gly-X-Y triple sequence required for the normal formation of stable triple helical type II collagen molecules resulting in the complete absence of type II collagen in the cartilage, which had a gelatinous composition [11]. This type II collagen is essential for normal development of bones and other connective tissue that form the body's supportive framework [9].

The children with achondrogenesis are usually born premature, stillborn or die in the neonatal period mostly from respiratory failure [1] [12]. The incidence is rare, most cases reported are delivered as stillbirth. Type I achondrogenesis is an autosomal recessive disorder, the risk of having another affected child is $25 \%$ for couple who had a first child with this condition [7]. Type II incidence rate is approximately $1 / 40,000-1 / 60,000$ births [1] [6].

We reported this case study of a live term newborn infant with achondrogenesis type II.

\section{Case Report}

An 11 hours old term male neonate was admitted into the Special Care Baby 
Unit (SCBU) of the Federal Medical Centre, Yola with complaints of abnormal shortening of both upper and lower limbs, and left hemi-scrotal swelling since birth.

Patient is a product of term gestation, booked at a secondary health Centre but delivered at home via spontaneous vaginal delivery, and baby said to have not cried immediately after birth, however ApgarScores could not be ascertained. No family history of congenital malformation, fetal wastage or maternal exposure to radiation or chemical/traditional concoction. Mother denied use of un-prescribed medications. There was history of whitish vaginal discharge at $3^{\text {rd }}$ trimester, no history of maternal febrile illness in pregnancy, and no history suggestive of polyhydramnios. Had tetanus toxoid (TT) given, intermittent presumptive therapy (IPT) and prescribed hematinics.

Mother is a 24-year-old housewife, while father is a 28 -year-old butcher in a monogamous family setting; both parents have no formal education and were displaced from their native home due to insurgency.

Examination revealed a uniformly pink baby, febrile $\left(39.1^{\circ} \mathrm{C}\right)$, was anicteric, cyanosed, with edematous lower limbs, he weights $2 \mathrm{~kg}$ and was noticed to have severe micromelia. Head circumference $35 \mathrm{~cm}$, had prominent forehead and depressed nasal bridge, micrognathia, low set ears, poor incurving of the pinna, skull is soft (craniotabes) with absence of bone at the temporal region, anterior fontanelle was widely patent with sutural diathesis. He was conscious with slightly depressed cortical stimuli and primitive reflexes (Moro, sucking and palmar reflexes).

Musculoskeletal system (MSS): the limbs were severely shortened with severe micromelia (Figure 1, Figure 2).

Respiratory system (RS): patient was dyspneic and tachypneic, RR 80 CPM, breath sound was broncho vesicular.

Heart rates 204 BPM, heart sounds were 1 and 2 only, no murmur heard.

Abdomen was full, moved with respiration, umbilical stump tied with thread and clean, had normal male external genitalia, there was left direct inguinal hernia and absent testicles.

At admission, a working diagnosis of multiple skeletal dysplasia and presumed sepsis was made. An x-ray done reveals normal cranial ossification with short ribs and failure of ossification of lumbar and cervical spines, sacrum and pubic bones necessitating a change of diagnosis to Achondrogenesis type II with presumed sepsis (Figure 3, Figure 4).

Full blood Count reveals a PCV of $30 \%$, WBC of $19.9 \times 10^{9} / 1$, neutrophil $46 \%$, lymphocyte $54 \%$, platelets $247 \times 10^{9} / 1$ and left shift neutrophils with toxic cytoplasmic changes. Other investigations (E/U/Cr and Blood culture) could not be done due to some technical factors.

$\mathrm{He}$ was placed on intranasal $\mathrm{O}_{2}$, parenteral cefuroxime, gentamicin and calcium gluconate. Baby also had blood transfused at $15 \mathrm{mls} / \mathrm{kg}$ of packed cells on account of severe anaemia, IV frusemide given as $1 \mathrm{mg} / \mathrm{kg}$ at commencement 


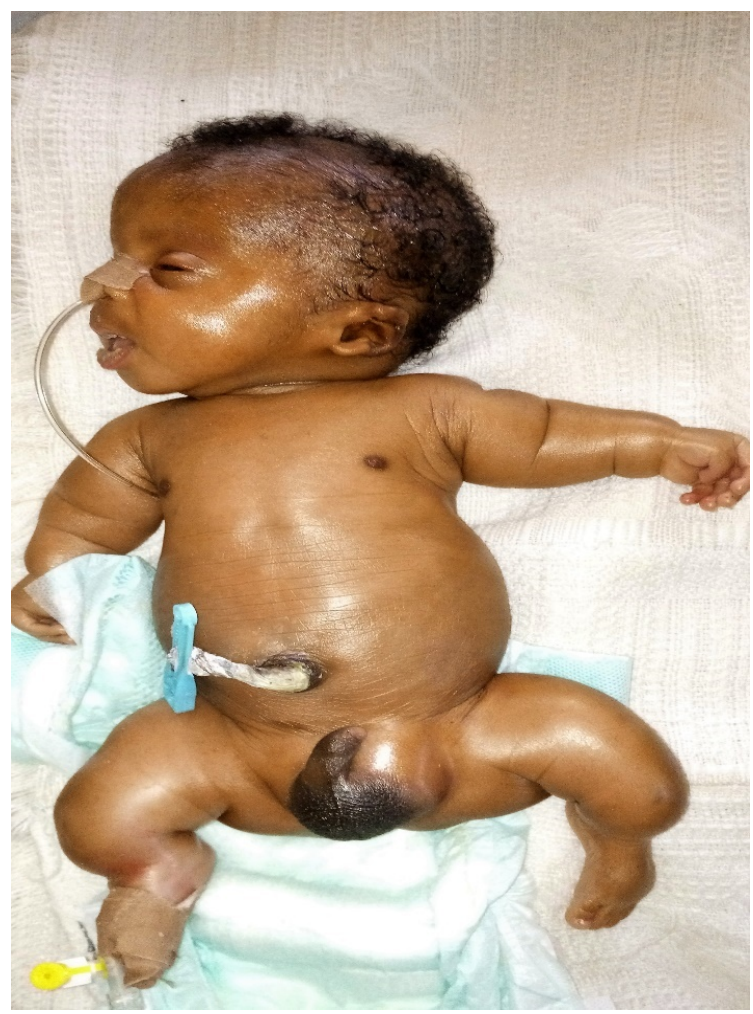

Figure 1. An infant with type II achondrogenesis showing extremely short stature with short limbs and flexion contractures at knee.

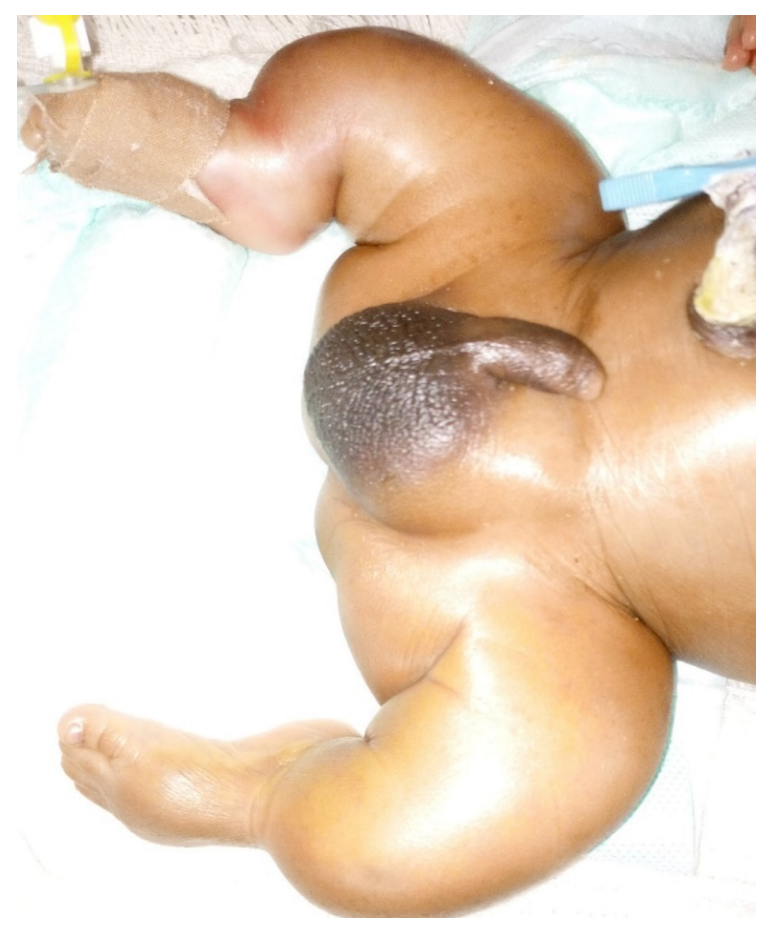

Figure 2. An infant with achondrogenesis type II showing short lower limbs and flexion contractures at knees. 


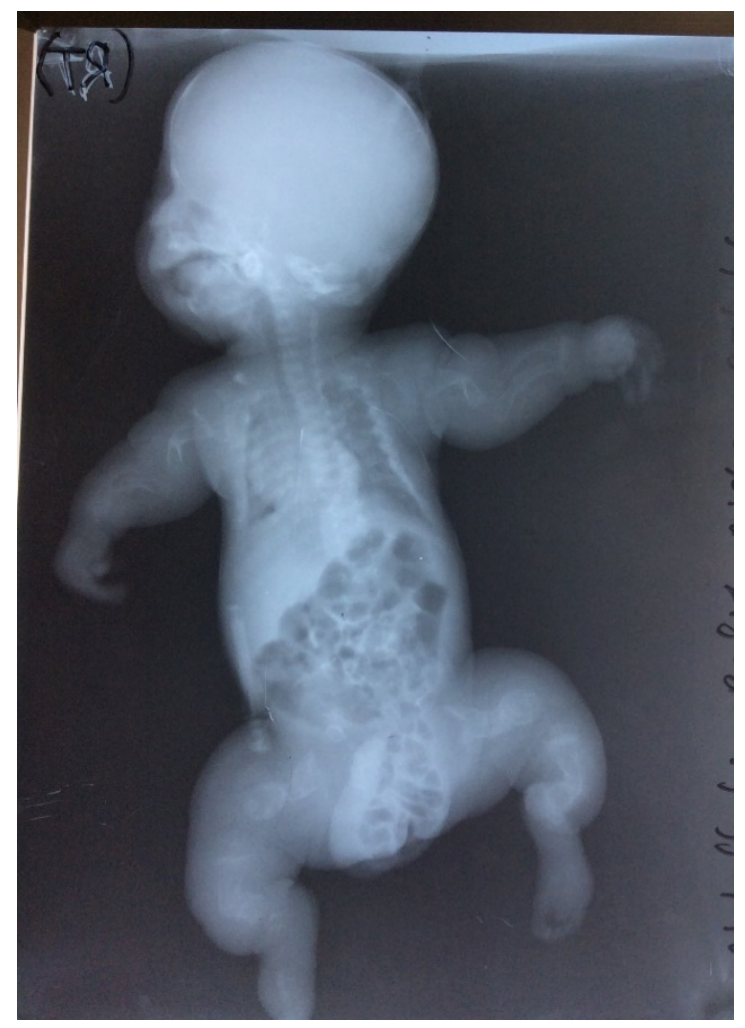

Figure 3. Showing normal cranial ossification with short ribs and failure of ossification of lumbar and cervical spines, sacrum and pubic bones.

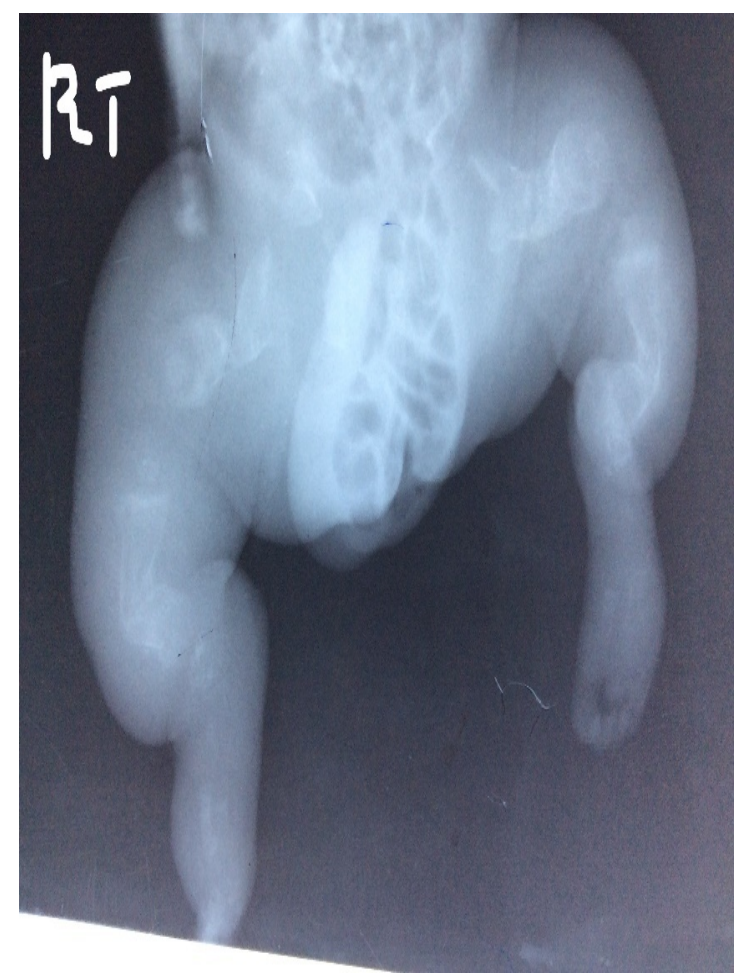

Figure 4. Showing failure of ossification of sacrum and pubic bones with. 
of blood transfusion. At 72 hours of life. Baby's clinical condition improved slightly evidenced by lowering of respiratory rate and heart rate. However, within the next 24 hours, baby's clinical condition worsened with baby gasping. Efforts at resuscitation were not successful and baby was certified dead at age 5 days.

\section{Discussion}

Achondrogenesis type II is one of the severe forms of congenital achondroplasia where there is malformation of bone and cartilage which are very rare with few cases reported and mostly as stillbirth. It is inherited as an autosomal dominant disorder; a result of mutation of COL2A1 gene, chromosomal locus 12q8.11 q13.2, a collagenopathy type II with sporadic cases [5].

The recurrence risk of having another child with achondrogenesis for two in unaffected parents is rare. However, the incidence of achondrogenesis type I (autosomal recessive) is 25\%. There is no record of sex preponderance, however most reported cases are male hence the risk of high mutation rate in male because of the germ-cell division rate is higher than that of female. Achondrogenesis type II is probably a disorder representing degeneration of proliferating epiphyseal cells with extremely deficient matrix. It has hypercellular cartilage with markedly deficient cartilaginous matrix, primitive mesenchymal chondrocytes with abundant clear cytoplasm [6].

The vertebral bodies in achondrogenesis are often completely absent or only barely visible on roentgenograms. The vertebral bodies in the lower thoracic and upper lumbar spine, the first to ossify in the normal infant are usually the most clearly seen. The femora are shortened, but usually not bowed. The metaphyseal margins are irregular and not clearly outlined. Projection of periosteal ossification and the margins of the ends of the bones may appear as faint striations. The humeri are short with prominently widening end [12].

Danny Cham et al. reported a case of achondrogenesis type II in 1994 and published in 1995 that it was caused by heterogenous mutation of COL2AI gene that resulted in the substitution of $\mathrm{Gly}^{769}$ by Ser in the triple helical domain of aI(II) chain, concluded that the mutations of COL2A1 resulted in the absence of type II collagen in the cartilage matrix caused achondrogenesis type II [11].

Kim et al. reported the first case in a Korean child, the fetus was at 29-week gestational age which had about $1 / 3$ of the expected length of lower limbs (length $5.5 \mathrm{~cm}$ as against $14 \mathrm{~cm}$ ), in another report; Lee and colleagues reported the $2^{\text {nd }}$ case of achondrogenesis type II in Korea which recorded about 1/5 of the expected length $(5 \mathrm{~cm}$ as against $10 \mathrm{~cm}$ ).

Rajeswari et al. reported a case of female fetus in 2015 delivered at 5 months gestation by termination of pregnancy following prenatal sonographic examination [13]. Other reported cases were diagnosed prenatally using sonological examination which can be detected as early as 12 weeks of gestational age. It is also of importance to differentiate this rare lethal fetal dwarfism from other forms of 
chondroplasia such as thanatophoric syndrome and achondroplasia which are less lethal.

Evidence based diagnostic approach can be done by prenatal sonological examination, infantogram, histopathological study, DNA analysis, mutational analysis and by chondrocyte culture [14], however, most of these investigations are not readily available in developing countries (Nigeria inclusive) and limited health centers assessable to pregnant mothers, thereby making diagnosis difficult in early pregnancy in developing countries like ours.

In our case, although the pregnancy was booked at a secondary health facility, the diagnosis was missed prenatally and the pregnancy was carried to term unlike others ending up as preterm delivery. The woman could have benefited from termination of pregnancy at early stage, had this been diagnosed early. Our patient presented at age 11 hours with normal cranial ossification, however, has failed ossification in the cervical and lumbar spines. The baby also has failure of ossification in the sacrum and pubic bones in addition to features of sepsis compounded by severe anaemia worsening the outcome. We report this case to remind clinicians of the occurrence of this rare form of achondroplasia-achondrogenesis type II and a need to carry out early sonological examination of all pregnant mothers.

\section{Conclusion}

Achondrogenesis type II is a lethal form of skeletal dysplasia that is inherited as a de novo autosomal dominant mutation of COL2A1 gene on chromosome $12 \mathrm{q} 8.11$ - q13.2. Whenever a skeletal dysplasia in a fetal dwarfism is suspected, a proper work-up plan should be done to evaluate family history. A clinical, radiographic and histopathologic examination, should be done and confirmed by genetic study. Efforts should be made to differentiate this lethal dysplasia from others which are less lethal such as thanatophoric dwarfism and achondroplasia. Following evidence-based diagnosis, patients could be offered termination of pregnancy after counseling.

\section{Consent}

An informed consent was obtained from the patient's parents.

\section{References}

[1] Verma, A. (2008) Achondrogenesis: Spotters in Pediatrics. Jaypee Brothers Medical Publishers Ltd, New Delhi, 186-187.

[2] Yadav, A. and Yadav, M. (2014) Achondrogenesis: A Lethal Chondrodystrophy. IJSS Case Reports and Reviews, 1, 1-3.

[3] Favre, L. and Cormier-Daire, V. (2003) Achondrogenesis. Orphaned Encyclopedia. http://www.orpha.net.fr/data/patho/GB/uk-achondro.pdf

[4] Chan, D., Cole, W.G., Chow, C.W., Mundlos, S. and Bateman, J.F. (1995) A COL2A1 Mutation in Achondrogenesis Type II Results in the Replacement of Type II Collagen by Type I and III Collagens in Cartilage. Journal of Biological Chemi- 
stry, 270, 1747-1753. https://doi.org/10.1074/jbc.270.4.1747

[5] Saldino, R.M. (1971) Lethal Short-Limbed Dwarfism: Achondrogenesis and Thanatophoric Dwarfism. https://www.ajronline.org/

[6] Genetic Home Reference (2008) Achondrogenesis. https://ghr.nlm.nih.gov/condition/achondrogenesis

[7] Swarr, D.T. and Sutton, V.R. (2018) Skeletal Dysplasias in the Newborn: Diagnostic Evaluation and Developmental Genetics.

http://neoreviews.aappublications.org/cgi/content/full/neoreviews

[8] Chen, H. (2013) Achondrogenesis. https://emedicine.staging.medscape.com/article/941176-overview

[9] Lee, H.S., Doh, J.W., Kim, C.J. and Chi, J.G. (2000) Achondrogenesis Type II (Langer-Saldino Achondrogenesis): A Case Report. Journal of Korean Medical Science, 15, 604-608. https://doi.org/10.3346/jkms.2000.15.5.604

[10] Jones, K.L. (2006) Achondrogenesis: Smith's Recognizable Patterns of Human Malformation. 6th Edition, Elsevier Saunders Publisher, Philadelphia, PA.

[11] NORD (National Organization for Rare Disorder (2017) Achondrogenesis. https://rarediseases.org/rare-disease/achondrogenesis

[12] Ramya Shree, T.R. and Saraswathi, K. (2015) A Case Report of Antenatal Diagnosis of Achondrogenesis Type I. Research Journal of Pharmaceutical, Biological and Chemical Science, 6, 551-555.

[13] Rajeswari, R., Rajani, V. and Rama Reddy, B.V. (2015) A Rare Case Report of Fetal Autopsy-Achondrogenesis Type II (Langer-Saldino). Paripex-Indian Journal of Research, 4, 103-104.

[14] Chem, H. (2006) Achondrogenesis: Atlas of Genetic Diagnosis and Counseling. Humana Press Inc, New York, 7-9. 